\title{
Lights and shadows of NSAIDs in bone healing: the role of prostaglandins in bone metabolism
}

This article was published in the following Dove Press journal:

Drug Design, Development and Therapy

\author{
Barbara Lisowska' \\ Dariusz Kosson ${ }^{2}$ \\ Karolina Domaracka ${ }^{3}$ \\ 'Department of Anesthesiology and \\ Intensive Care, John Paul II Western \\ Hospital in Grodzisk Mazowiecki, \\ Grodzisk Mazowiecki, Poland; \\ ${ }^{2}$ Division of Teaching, Department \\ of Anaesthesiology and Intensive \\ Care, Medical University of Warsaw, \\ Warsaw, Poland; ${ }^{3}$ Department of \\ Anaesthesiology and Intensive \\ Care, Medical University of Warsaw, \\ Warsaw, Poland
}

\begin{abstract}
In this review, we discuss the current data about the anatomy and function of bone tissue with particular regard to influence of prostaglandins. Bone tissue dynamics are characterized by a constant remodeling process that involves all bone tissue cells. The communication between bone component cells and other organs is necessary for bone remodeling equilibrium and confirms the dynamic character of bone tissue. Remodeling is also a vital element of healing processes and in adapting bone tissue to stress responses. Therefore, in our review we present the role and significance of bone cells and signaling pathways enabling maintenance of bone homeostasis and remodeling process stability. Cyclooxygenase (COX) is a crucial enzyme in the production of prostaglandins and thromboxane. We focus on the role of COX isoenzymes with highlighting their connection with bone formation, resorption and repair. Prostaglandins are known as arachidonic acid metabolites acting through specific membrane receptors and play an important role in the regulation of osteoblast and osteoclast functions. Prostaglandin PGE2 with its four defined receptors (EP1R, EP2R, EP3R and EP4R) is crucial to maintain balanced bone turnover. Their stimulatory or inhibitory effects appear to depend on different structure-activity relations and signaling pathways. We have described the role of these receptors in bone metabolism and healing. We conclude that the activity of prostaglandins in bone tissue is defined by maintaining bone remodeling balance and its reactions to humoral mediators and mechanical stress. Most data confirm that among prostaglandins, PGE2 takes part in all processes of trauma response, including homeostasis, inflammation and healing, and plays a key role in bone physiology.
\end{abstract}

Keywords: bone cells and metabolism, COX, prostaglandins

\section{Introduction}

The biological processes of bone metabolism are characterized by a constant remodeling process that involves all bone tissue cells and it is known as a chain of biological events concerning both intracellular and extracellular molecular signaling pathways. They are regulated by many factors among which prostaglandins mediate the regulation of osteoblast and osteoclast functions by acting though specific membrane receptors. Prostaglandins are enzymatically derived metabolites of arachidonic acid that are implicated in bone remodeling, thus acting as a vital element of healing processes and in adapting bone tissue to stress responses. Among all prostaglandins, PGE2, in particular, is involved in widespread regulation of various processes of inflammation and bone metabolism, among other things. PGE2 acts through four G protein-coupled receptor subtypes present in bone cells.

The aim of this review is the presentation of current data about the anatomy and function of bone tissue with particular regard to influence of prostaglandins in various processes of bone metabolism.
Correspondence: Dariusz Kosson Division of Teaching, Department of Anaesthesiology and Intensive Care, Medical University of Warsaw,

Warsaw, Poland

Email kosson@wp.pl
Drug Design, Development and Therapy 2018:12 1753-1758

(c) (1) (8) ๑ 2018 Lisowska et al. This work is published and licensed by Dove Medical Press Limited. The full terms of this license are available at https:/www.dovepress.com/terms.php cc) hereby accept the Terms. Non-commercial uses of the work are permitted without any further permisision from Dove Medical Press Limited, provided the work is properly attributed. For permission for commercial use of this work, please see paragraphs 4.2 and 5 of our Terms (https://www.dovepress.com/terms.php). 


\section{Bone tissue}

Bone tissue is characterized by a wide array of reactions to mechanical, metabolic, inflammatory and hormonal stimuli. Its ability to adapt to various conditions, especially mechanical demands and fatigue loading changes, is certainly worth mentioning. Bone tissue plays a role in many processes and performs multiple functions, including locomotion, skeletal support and protection of vital organs. Moreover, bone tissue is the primary storehouse for calcium and phosphorus, takes part in maintaining electrolyte balance and protects vital organs by accumulating heavy metals. ${ }^{1}$ Hematopoiesis, T-lymphocyte differentiation, adult neutrophil elimination and the triggering of inflammatory processes in response to pathological conditions occur in bone marrow.

From a histological point of view, bone tissue is a type of connective tissue. Bone tissue consists of cells surrounded by an extracellular matrix, which contains organic and inorganic components. The organic part comprises collagen proteoglycans, which are non-mineralized and are primarily type 1 collagen. The inorganic part is mineralized and comprises calcium carbonates and calcium phosphates in the form of hydroxyapatite crystals. ${ }^{1}$ Osteogenic cells are typically found in bone tissue and include osteogenic cell precursors from various stem cells lineage, osteoblasts, osteocytes and osteoclasts. Osteoblasts have cuboidal shapes and are found near bone surfaces. Osteoblasts are mature bone-forming cells responsible for bone formation by producing bone matrix proteins and play an essential role in bone remodeling. Osteoblasts express several receptors sensitive for hormones, which primarily include parathyroid hormone $(\mathrm{PTH})$ and estrogen and 1,25-dihydroxyvitamin D3 [1.25(OH)2D3] and other local factors, such as cytokines and prostaglandins. In the presence of these hormones, osteoblasts secrete proteins and proteoglycans, which have a core protein and side chains. Among collagen proteins, collagen type 1 is the most important. Examples of non-collagen proteins include osteonectin, bone sialoprotein and osteopontin. The mature forms of osteoblast can be subjected to apoptosis or undergo transformation into osteocytes or bone-lining cells., ${ }^{2,3}$

Surrounded by a cellular matrix, osteoblasts transform into osteocytes, the role of which in bone homeostasis has not been fully elucidated. Osteocytes respond to mechanical loading stimuli by secreting several factors, such as prostaglandins, ATP, nitric oxide and Wnt1 proteins, ie, critical molecules that can modulate the transformation of osteoblasts and osteoclasts. Wnt1 proteins belong to a group of glycoproteins that activate Wingless (Wnt) signaling pathways in the cytoplasm and cell nucleus by enhancing the expression of target genes with autocrine, endocrine and paracrine effects. In response to Wnt signaling, osteocytes release sclerostin. By binding to low-density lipoprotein receptorrelated protein-5/6, the complex acts as a $\mathrm{Wnt} / \beta$-catenin signaling pathway inhibitor. The Wnt signaling pathway is crucial for creating bone density. Animals with Wnt mutations have been shown to be prone to bone fragility due to limited osteoblast activity from the loss of the Wnt1 signaling pathway in osteocytes and osteoblasts. Likewise, pathway disorders and genetic mutations in bone tissue target cells have resulted in decreased bone mass and increased bone fragility. Moreover, osteocytes are known as the main source of receptor activator of nuclear factor- $\mathrm{\kappa B}$ ligand (RANKL). ${ }^{46}$ In reference to osteoclasts, osteocytes act through changes to RANKL expression.

In contrast to osteoblasts, which originate from mesenchymal stem cells, osteoclasts originate from mononuclear cells of hematopoietic stem cells. Their differentiation is controlled by two key cytokines, RANKL and macrophage colonystimulating factor (M-CSF), released from osteoprogenitor cells and osteoblasts. The stimulation of c-Fms receptors for $\mathrm{M}-\mathrm{CSF}$, receptor activator of nuclear factor- $\mathrm{\kappa B}$ (RANK) and osteoclast-associated immunoglobulin-like receptor on osteoclast progenitors increases the expression of genes stimulating their differentiation. ${ }^{7}$ In inflammatory diseases, TNF- $\alpha$ can promote osteoclastogenesis responsible for osteolysis or bone density disturbance. The proliferation of osteoclasts is inhibited by osteoprotegerin (OPG), which prevents RANK/ RANKL connection, and by calcitonin, which acts through calcitonin receptors. ${ }^{8-10}$ The main function of osteoclasts in bone remodeling is initiating bone resorption.

Bone-lining cells are quiescent, flat-shaped, mature osteoblasts located on the bone surface. They seem to play a role in bone remodeling by participating in osteoclastogenesis and synthesizing messengers, such as OPG and RANKL. ${ }^{3}$

\section{Bone remodeling}

Bone tissue dynamics are characterized by a constant remodeling process that involves five phases: activation, resorption, reversal, formation and mineralization. Figure 1 shows the phases of remodeling process.

Beginning with resorption by clusters of bone-resorbing osteoclasts and ending with the formation of new bone tissue by osteoblasts, this process occurs in special complexes called basic multicellular units (BMUs). BMUs coordinate the participation of traditional bone cells in bone turnover. ${ }^{11}$ Remodeling is a vital element of healing processes and in 


\section{The bone remodeling process}

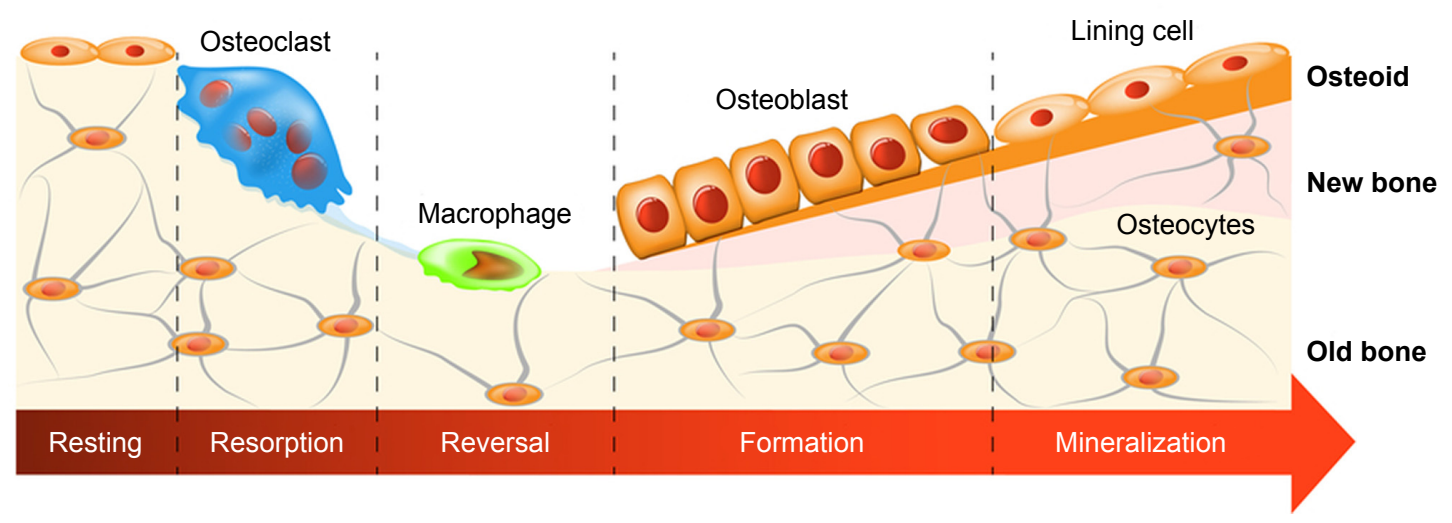

Figure I Remodeling process is characterized by five phases: resting, resorption, reversal, formation and mineralization.

adapting bone tissue to stress changes. A balance between resorption and bone formation ensures correct bone tissue function and is dependent on many local system factors that regulate bone cell functions by cell surface receptor connections. Studies have shown that in addition to primary bone cells, neutrophils and B and T lymphocytes also participate in bone remodeling; their participation was found to be dependent on local and systemic mediators, such as prostaglandins, cytokines, growth factors, Wnt signaling, bone morphogenetic proteins (BMPs), hormones and 1,25(OH)2D3.,.$^{3,12}$ Intercellular communication is vital to remodeling because it maintains the balance between these two processes. Osteoblast-osteoclast communication is established through cell-cell contacts by the binding of RANK ligands or OPG to RANK receptors in osteoclasts. The RANKL/RANK/ OPG pathway is regulated by the aforementioned local and systemic factors. Cell-cell and cell-extracellular matrix interactions can occur due to paracrine factors released from osteoblasts. ${ }^{7,13,14}$ Another example that ensures communication, balanced remodeling and external signal responses is the GAP conjunction, which is formed by proteins known as connexins. The distribution of connexins determines the conjunction channel type and its properties. A connexin of great significance is connexin 43, which with fibroblast growth factors (FGFs) regulates osteoblast transformation in response to mechanical stimulation. Intercellular exchanges through GAP are regulated by anabolic and catabolic bone metabolism factors, such as PTH, BMP-2 and tumor grown factor $\beta$. Furthermore, GAP and PGE2 connections are vital to mechanical bone elasticity. Osteoblasts and osteocytes can also communicate with each other by means of cytoplasmic connections or interact on cell surfaces. ${ }^{15,16}$ All processes involved in bone remodeling play critical roles in bone healing, skeletal adaptation to biomechanical forces and calcium balance. It is worthwhile to highlight that all described processes are related with bone remodeling under physiological conditions.

\section{Prostaglandin synthesis}

Prostaglandins, prostacyclin (PGI2) and thromboxane A2 (TXA2) are known as prostanoids and are arachidonic acid metabolites released from cells under the influence of many factors that act though specific membrane receptors. Growth factors (eg, FGF, IFG and PDGF), hormones (vasopressin) and neurotransmitters (NA and $\mathrm{ACh}$ ) are among those factors. Arachidonic acid transformation is regulated by two cyclooxygenase (COX) isoenzymes, COX-1 and COX-2. Due to subsequent cyclooxygenation and peroxygenation, unstable prostaglandins PGG2 and PGH2 are formed. Next, these prostaglandins undergo further transformation in the presence of specific prostanoid synthases. The final transformation substrates are the prostaglandins PGE2, PGF2 and PGD2; PGI2; and TXA2. ${ }^{17-19}$ The prostanoid synthesis profile is dependent on differences in enzymatic expression in cells under inflammation. Macrophages, for instance, release PGE2 and TXA2, whereas mast cells release prostaglandin PGD2. ${ }^{20}$

Prostaglandins play key roles in trauma response. For example, PGE2 and PGI2 have significant vasodilatory properties, whereas platelet-released TXA2 is known as a vasoconstrictor that increases platelet aggregation.

We shall now discuss three COX isoenzymes: COX-1, COX-2 and COX-3. Although these enzymes are found in their physiological state, COX-1 plays a major role in regulating the functions of many organs, whereas COX-2 is more notable in trauma, inflammation and proliferative 
disorders. ${ }^{21}$ COX-3 constitutes a COX-1 subcategory of a different amino-acid sequence and has characteristic sensitivity to acetaminophen. Acetaminophen is known to have similar analgesic and antipyretic properties to NSAIDs but does not have any anti-inflammatory effects due to its poor ability to inhibit inflammation-induced COX. ${ }^{22}$ Furthermore, acetaminophen does not affect platelets and the GI tract. COX -3 can be found in the hippocampus and microglia cells. ${ }^{22,23}$ Recent findings have revealed structural similarities between acetaminophen and benzodiazepine receptors, ie, integral parts of $\mathrm{GABA}_{\mathrm{A}}$ receptors. ${ }^{24}$

\section{The role of prostaglandins in bone metabolism}

Although COX-1 and COX-2 affect the properties of bone tissue, COX-2 is dominant in osteoblasts. The studies with COX-1 or COX-2 knockout animals have confirmed a crucial role of COX-2 in maintaining bone density and strength, PTH levels and renal function. COX-2 knockout animals showed significantly lower bone density and upregulated PTH compared with control groups. Furthermore, in COX-2 knockout animals, a shortened duration of survival and renal failure with elevated calcium and 1,25(OH)2D3 levels were observed. In COX-1 knockout animals, bone density improved, but the levels of calcium and PTH in serum were comparable with those of control group. These changes in the COX-2 knockout animals can be interpreted as compensatory reactions for a lack of COX-2. ${ }^{25,26}$ Animal testing has also defined the role of COX-2 in adapting bone tissue to fluid shear stress. ${ }^{27}$

Prostaglandin receptors are G-protein coupled receptors in the A-rhodopsin-like receptor superfamily. In bone tissue, the function of prostaglandins is regulated via their receptors for ensuring bone turnover balance. The influence on bone cell differentiation by prostaglandins is dependent on the activation of their various structures through different signaling pathways. Prostaglandin PGE2 with its four defined receptors (EP1R, EP2R, EP3R and EP4R) is crucial to maintaining balanced bone turnover. ${ }^{28,29}$ The action of prostaglandin E2 (mediated mainly through EP2R and EP4R) causes increased cyclic AMP, which enhances its signal by activating protein kinase A and cAMP-dependent ion channels. Cyclic AMP and COX induction are better stimulated by PGE2 through binding to the EP2 receptor than to EP4R receptor.

Furthermore, through the EP2 receptor, PGE increases the expression of c-Fos transfusion factor, which controls the differentiation of osteoblasts and osteoclasts. The PGE2 anabolic effect is also stimulated by PGE2 and PGE4 receptors through the stimulation of the mitogen-activated protein kinase signaling pathway. Binding PGE2 to EP4R by means of increasing BMP-2 and RANKL expression improves bone healing and regeneration..$^{30,31}$

However, binding PGE2 to EP3R provides an opposite effect due to the inhibition of adenyl cyclase and limited cAMP synthesis. Upon the binding of PGE2 to EP1R, phospholipase $\mathrm{C}$ is activated, which contributes towards enhanced $\mathrm{Ca}^{++}$influx due to protein kinase $\mathrm{C}$ activity. ${ }^{19,32}$ Binding PGE2 to EP1R downregulates bone formation. Studies of animals lacking the EP1 receptor showed better bone healing and higher bone volume and strength. The loss of the EP1 receptor was hypothesized to improve osteoblastic differentiation by increasing oxygen consumption. ${ }^{33}$

Other animal tests have substantiated the effect of prostaglandins on bone turnover. However, test results have not conclusively shown their advantage over osteoclasts or osteoblasts. PGE2 administration contributed to increased bone mass, bone stiffness and mechanical strength in animals. However, the growth of long bones was also reduced. ${ }^{34,35}$

The main effect that PGE2 has on bone resorption is induced by activating the NF- $\kappa \mathrm{B}$ ligand (RANKL) on precursors of osteoclastic cells. Moreover, PGE2 overexpresses M-CSF, enabling it to bind to the c-Fms receptor on the surface of osteoclast precursor cells necessary for their proliferation. ${ }^{19}$

The osteoclast precursors express four receptor subtypes, EP1R-EP4R, for PGE2. Only the EP1R receptor is localized in mature osteoclasts. The role of individual receptors in bone resorption has been demonstrated in tests involving their agonists. Test results indicated that EP4R agonists play higher roles than other agonists. EP2R agonists are only partially involved, whereas EP1R and EP3R agonists have no effects on osteoclast formation. Moreover, studies of EP2R knockout mice have shown responses to the stimulation of EP2R by PGE2, wherein the level of serum calcium increased. ${ }^{36,37}$ Findings from Kobayashi et al suggested that the induction of osteoclast differentiation is initiated by PGE2 through EP4 and EP2. The downregulation of PGE2 through both receptors was also confirmed. ${ }^{38}$

The administration of PGE2 activates bone formation and resorption signaling pathways. PGE2 administration techniques also are of importance. The continuous administration of PGE2 induces resorption, whereas intermittent PGE2 has an anabolic effect. The animal study performed by Tian et al showed that PGE2, given continuously, significantly stimulated bone resorption contributing to the decrease in cortical bone mass and negative bone balance while the 
intermittent administration of PGE2 activated bone formation with an increase in bone mass and its thickness. In both cases, the changes were dependent on the given dose. In addition, the anabolic effect of intermittent PGE2 is similar to result of PTH given in the same way. The beneficial skeletal changes associated with intermittent PTH administration were confirmed by two studies. ${ }^{39,40}$

Bone trauma can contribute towards local hypoxia. Tissue hypoxia has been known to stimulate PGE2 production, whereas, apart from COX-1 and COX-2, the signaling pathway of hypoxia-inducible factor (HIF) is significant, especially the HIF- $1 \alpha$ subunit, in osteoblasts. Thus, the release pathway of PGE under hypoxic conditions has been determined. ${ }^{41}$

Prostaglandins do not accumulate in cells, and their degradation to 15-keto-prostaglandins is NAD ${ }^{+} 15$-hydroxyprostaglandin dehydrogenase-induced (15-HPGD). Research has shown that the inhibition of 15-HPGD may contribute to the PGE2 upregulation, which, given a very short PGE2 half-life, may prolong its benefits. ${ }^{42}$

In this manuscript the authors focused on the influence of prostaglandins on the osteogenic cells and physiology of bone tissue in order to emphasize the negative effects of their blockade by NSAIDs. The role of NSAIDs on presented cells of bone tissue and all stages of bone healing will be discussed in the manuscript titled Positives and negatives of nonsteroidal anti-inflammatory drugs in bone healing: the effects of non-steroidal anti-inflammator.

\section{Conclusion}

The activity of prostaglandins in bone tissue is defined by maintaining bone turnover balance and its reactions to humoral mediators and mechanical stress. A balanced osteoblast and osteoclast activity guarantees bone turnover equilibrium. PGE2 takes part in all processes of trauma response, including homeostasis, inflammation and healing, and plays a key role in bone physiology.

\section{Disclosure}

The authors report no conflicts of interest in this work.

\section{References}

1. Amini AR, Laurencin CT, Nukavarapu SP. Bone tissue engineering: recent advances and challenges. Crit Rev Biomed Eng. 2012;40(5):363-408.

2. Neve A, Corrado A, Cantatore FP. Osteoblast physiology in normal and pathological conditions. Cell Tissue Res. 2011;343(2):289-302.

3. Florencio-Silva R, Rodrigues da Silva Sasso G, Sasso-Cerri E, Simões MJ, Cerri PS. Biology of bone tissue: structure, function, and factors that influence bone cells. Biomed Res Int. 2015;2015:421746.

4. Rauch F. The brains of the bones: how osteocytes use WNT1 to control bone formation. J Clin Invest. 2017;127(7):2539-2540.
5. Joeng KS, Lee YC, Lim J, et al. Osteocyte-specific WNT1 regulates osteoblast function during bone homeostasis. J Clin Invest. 2017;127(7): 2678-2688.

6. Xiong J, Piemontese M, Onal M, et al. Osteocytes, not osteoblasts or lining cells, are the main source of the RANKL required for osteoclast formation in remodeling bone. PLoS One. 2015;10(9):e0138189.

7. Matsuo K, Irie N. Osteoclast-osteoblast communication. Arch Biochem Biophys. 2008:473(2):201-209.

8. Clarke B. Normal bone anatomy and physiology. Clin J Am Soc Nephrol. 2008;3(Suppl 3):S131-S139.

9. Peruzzi B, Teti A. The physiology and pathophysiology of the osteoclast. Clin Rev Bone Miner Metab. 2012;10(2):71-97.

10. Teitelbaum SL. Osteoclasts: what do they do and how do they do it? Am J Pathol. 2007;170(2):427-435.

11. Raggatt LJ, Partridge NC. Cellular and molecular mechanisms of bone remodelling. J Biol Chem. 2010;285(33):25103-25108.

12. Xiao W, Wang Y, Pacios S, Li S, Graves DT. Cellular and molecular aspects of bone remodeling. Front Oral Biol. 2016;18:9-16.

13. Nakahama K. Cellular communications in bone homeostasis and repair. Cell Mol Life Sci. 2010;67(23):4001-4009.

14. Crane JL, Cao X. Bone marrow mesenchymal stem cells and TGF- $\beta$ signaling in bone remodelling. $J$ Clin Invest. 2014;124(2):466-472.

15. Cherian PP, Cheng B, Gu S, Sprague E, Bonewald LF, Jiang JX. Effects of mechanical strain on the function of Gap junctions in osteocytes are mediated through the prostaglandin EP2 receptor. J Biol Chem. 2003; 278(44):43146-43156.

16. Plotkin LI, Laird DW, Amedee J. Role of connexins and pannexins during ontogeny, regeneration, and pathologies of bone. BMC Cell Biol. 2016;17(Suppl 1):S19.

17. Burdan F, Chałas A, Szumiło J. Cyklooksygenaza i prostanoidyznaczenie biologiczne [Cyclooxygenase and prostanoids - biological implications]. Postepy Hig Med Dosw (Online). 2006;60: 129-141. Polish [with English abstract].

18. Fitzpatrick FA. Cyclooxygenase enzymes: regulation and function. Curr Pharm Des. 2004;10(6):577-588.

19. Blackwell KA, Raisz LG, Pilbeam CC. Prostaglandins in bone: bad cop, good cop? Trends Endocrinol Metab. 2010;21(5):294-301.

20. Ricciotti E, FitzGerald GA. Prostaglandins and inflammation. Arterioscler Thromb Vasc Biol. 2011;31(5):986-1000.

21. Kulkarni SK, Jain NK, Singh A. Cyclooxygenase isoenzymes and newer therapeutic potential for selective COX-2 inhibitors. Methods Find Exp Clin Pharmacol. 2000;22(5):291-298.

22. Kis B, Snipers J, Busija DW. Acetaminophen and the cyclooxygenase-3 puzzle: sorting out facts, fictions, and uncertainties. J Pharmacol Exp Ther. 2005;315(1): 1-7.

23. Botting R, Ayoub SS. COX-3 and the mechanism of action of paracetamol/acetaminophen. Prostaglandins Leukot Essent Fatty Acids. 2005;72(2):85-87.

24. Ahangar N, Esam Z, Bekhradnia A, Ebrahimzadeh MA. Hypothermic activity of acetaminophen; involvement of GABAA receptor, theoretical and experimental studies. Iran J Basic Med Sci. 2016;19(5): $470-475$.

25. Myers LK, Bhattacharya SD, Herring PA, et al. The isozyme-specific effects of cyclooxygenase-deficiency on bone in mice. Bone. 2006;39(5): $1048-1052$.

26. Xu M, Choudhary S, Goltzman D, et al. Do cyclooxygenase-2 knockout mice have primary hyperparathyroidism? Endocrinology. 2005; 146(4):1843-1853.

27. Stevens HY, Meays DR, Yeh J, Bjursten LM, Frangos JA. COX-2 is necessary for venous ligation-mediated bone adaptation in mice. Bone. 2006;38(1):93-104.

28. Raisz LG. Physiologic and pathologic roles of prostaglandins and other eicosanoids in bone metabolism. $J$ Nutr. 1995;125(7 Suppl): 2024S-2027S.

29. Hata AN, Breyer RM. Pharmacology and signaling of prostaglandin receptors: multiple roles in inflammation and immune modulation. Pharmacol Ther. 2004;103(2):147-166. 
30. Minamizaki T, Yoshiko Y, Kozai K, Aubin JE, Maeda N. EP2 and EP4 receptors differentially mediate MAPK pathways underlying anabolic actions of prostaglandin E2 on bone formation in rat calvaria cell cultures. Bone. 2009;44(6):1177-1185.

31. Markovič T, Jakopin Ž, Dolenc MS, Mlinarič-Raščan I. Structural features of subtype-selective EP receptor modulators. Drug Discov Today. 2017;22(1):57-71.

32. Sakuma Y, Li Z, Pilbeam CC, et al. Stimulation of cAMP production and cyclooxygenase-2 by prostaglandin E(2) and selective prostaglandin receptor agonists in murine osteoblastic cells. Bone. 2004; 34(5):827-834.

33. Feigenson M, Eliseev RA, Jonason JH, Mills BN, O’Keefe RJ. PGE2 receptor subtype 1 (EP1) regulates mesenchymal stromal cell osteogenic differentiation by modulating cellular energy metabolism. J Cell Biochem. 2017;118(12):4383-4393.

34. Suponitzky I, Weinreb M. Differential effects of systemic prostaglandin E2 on bone mass in rat long bones and calvariae. J Endocrinol. 1998; 156(1):51-57.

35. Ito H, Ke HZ, Jee WS, Sakou T. Anabolic responses of an adult cancellous bone site to prostaglandin E2 in the rat. Bone Miner. 1993;21(3): 219-236.
36. Suzawa $T$, Miyaura $C$, Inada $M$, et al. The role of prostaglandin E receptor subtypes (EP1, EP2, EP3, and EP4) in bone resorption: an analysis using specific agonists for the respective EPs. Endocrinology. 2000;141(4): 1554-1559.

37. Li X, Tomita M, Pilbeam CC, Breyer RM, Raisz LG. Prostaglandin receptor EP2 mediates PGE2 stimulated hypercalcemia in mice in vivo. Prostaglandins Other Lipid Mediat. 2002;67(3-4):173-180.

38. Kobayashi Y, Take I, Yamashita T, et al. Prostaglandin E2 receptors EP2 and EP4 are down-regulated during differentiation of mouse osteoclasts from their precursors. J Biol Chem. 2005;280(25):24035-24042.

39. Tian XY, Zhang Q, Zhao R, et al. Continuous infusion of PGE2 is catabolic with a negative bone balance on both cancellous and cortical bone in rats. J Musculoskelet Neuronal Interact. 2007;7(4):372-381.

40. Compston JE. Skeletal actions of intermittent parathyroid hormone: effects on bone remodelling and structure. Bone. 2007;40(6):1447-1452.

41. Lee CM, Genetos DC, Wong A, Yellowley CE. Prostaglandin expression profile in hypoxic osteoblastic cells. J Bone Miner Metab. 2010; 28(1):8-16.

42. Karna S. In-vitro wound healing effect of 15-hydroxyprostaglandin dehydrogenase inhibitor from plant. Pharmacogn Mag. 2017;13(Suppl 1): S122-S126.
Drug Design, Development and Therapy

\section{Publish your work in this journal}

Drug Design, Development and Therapy is an international, peerreviewed open-access journal that spans the spectrum of drug design and development through to clinical applications. Clinical outcomes, patient safety, and programs for the development and effective, safe, and sustained use of medicines are the features of the journal, which

\section{Dovepress}

has also been accepted for indexing on PubMed Central. The manuscript management system is completely online and includes a very quick and fair peer-review system, which is all easy to use. Visit http://www.dovepress.com/testimonials.php to read real quotes from published authors.

Submit your manuscript here: http://www.dovepress.com/drug-design-development-and-therapy-journal 